\title{
Early termination algorithm for SLM-based PAPR reduction schemes in OFDM systems
}

\author{
Jung Suk Joo ${ }^{1, \text { a) }}$ \\ ${ }^{1}$ Dept. of Electronics Engineering, Hankuk University of Foreign Studies, \\ 89 Wangsan-ri, Mohyeon-myeon, Cheoin-gu, Yongin-si, Gyeonggi-do, 449-791, \\ Korea \\ a)jjs@hufs.ac.kr
}

Abstract: Selected mapping (SLM) is one of the most widely used peakto-average power ratio (PAPR) reduction schemes-it selects and transmits a signal having the smallest PAPR among candidate signals by performing a full search (FS). In this Letter, a simple modification to reduce the computational complexity of SLM-based PAPR schemes is proposed that terminates searching process when it meets a candidate signal not causing a clipping, instead of having the smallest PAPR.

Keywords: PAPR, SLM, OFDM, early termination, clipping

Classification: Wireless Communication Technologies

\section{References}

[1] R.W. Bäuml, R.F.H. Fisher, and J.B. Huber, "Reducing the peak-to-average power ratio of multicarrier modulation by selected mapping," Elect. Lett., vol. 32, no. 22, pp. 2056-2057, Oct. 1996. DOI: 10.1049/el:19961384

[2] H. Breiling, S.H. Muller-Weinfurtner, and J.B. Huber, "SLM peak-power reduction without explicit side information," IEEE Commun. Lett., vol. 5, no. 6, pp. 239-241, June 2001. DOI: 10.1109/4234.929598

[3] D.-W. Lim, S.-J. Heo, J.-S. No, and H. Chung, "On the phase sequence set of SLM OFDM scheme for a crest factor reduction," IEEE Trans. Signal Process., vol. 54, no. 5, pp. 1931-1935, May 2006. DOI: 10.1109/TSP.2006.871979

[4] C.-P. Li, S.-H. Wang, and C.-L. Wang, "Novel low-complexity SLM schemes for PAPR reduction in OFDM systems," IEEE Trans. Signal Process., vol. 58, no. 5, pp. 2916-2921, May 2010. DOI: 10.1109/TSP.2010.2043142

[5] Y. Rahamatallah and S. Mohan, "Peak-to-average power ratio reduction in OFDM systems: a survey and taxonomy," IEEE Commun. Surveys Tuts., vol. 15, no. 4, pp. 1567-1592, 4th Quart. 2013. DOI: 10.1109/SURV.2013.021313.00164

\section{Introduction}

The orthogonal frequency division multiplexing (OFDM) is widely used in broadband wireless communication systems due to its robustness in frequency-selective fading environments. However, OFDM system has a relatively high PAPR since each OFDM symbol is formed by combining many sub-carrier components. 
In order to overcome this drawback, PAPR reduction schemes have been actively studied, and among them we will consider selected mapping (SLM)-based PAPR reduction schemes $[1,2,3,4,5]$ : for each information data sequence, a candidate signal having the smallest PAPR is selected and transmitted by using a full search (FS) in the set of candidate signals. Although SLM based schemes do not cause signal distortion, they suffer from more computational complexity due to a FS.

In this Letter, in order to reduce the computational complexity of SLM-based PAPR schemes, we will propose a simple algorithm which terminates searching process immediately when it meets a candidate signal whose PAPR is less than a pre-determined target PAPR threshold value; the target PAPR threshold will be set not to increase the clipping probability of the transmit power amplifier. It will be shown through computer simulations that we can control the trade-off between PAPR reduction performance and computational complexity by adjusting the threshold.

\section{SLM-based OFDM system}

Figure 1 shows the block diagram of the SLM-based OFDM system having $N$ subcarriers. For performing SLM, there is a pre-defined phase sequence set consisting of $U$ phase sequences, $\left\{\mathbf{P}_{i}=\left[P_{i}(0), P_{i}(1), \cdots, P_{i}(N-1)\right], i=0,1, \cdots, U-1\right\}$, where $P_{i}(k)=e^{j \phi_{i, k}}$. For a given data sequence $\mathbf{X}=[X(0), X(1), \cdots, X(N-1)], U$ candidate OFDM symbols, $\left\{\mathbf{x}_{i}=\left[x_{i}(0), x_{i}(1), \cdots, x_{i}(N-1)\right], i=0,1, \cdots, U-1\right\}$ are generated from $N$-point IFFT on $\left\{\mathbf{X} \otimes \mathbf{P}_{i}\right\}$ where $\otimes$ represents component-wise multiplication. Then, a candidate OFDM symbol having the smallest PAPR, $\mathbf{x}_{i_{o}}$ is selected by performing a FS: that is,

$$
i_{o}=\arg \min _{0 \leq i \leq U-1}\left[P A P R_{i}\right],
$$

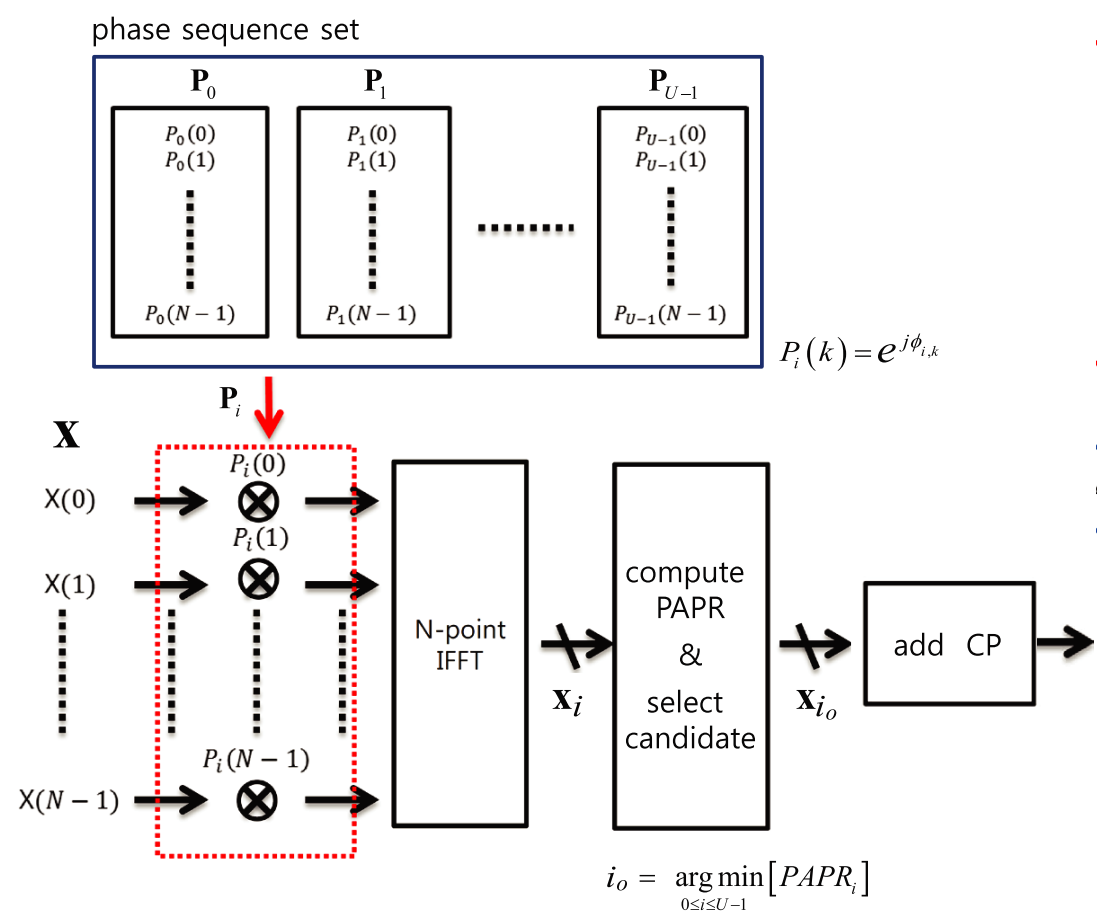


where

$$
P A P R_{i}=\frac{\max _{0 \leq k \leq N-1}\left[\left|x_{i}(k)\right|^{2}\right]}{\frac{1}{N} \sum_{k=0}^{k=N-1}\left|x_{i}(k)\right|^{2}} .
$$

It is clear that the computational complexity of SLM increases proportionally to the value of $U$.

In addition, $U$ has the value of $2^{L}$ in general, and $L$-bits information for indicating $i_{o}$ which is called side information (SI) should be transmitted to the receiver [5].

\section{Proposed early termination algorithm for SLM}

To characterize the peak power statistics of OFDM signal, the complementary cumulative distribution (CCDF) is generally used. The CCDF of PAPR is defined as the probability that an OFDM symbol has PAPR above a particular level $\gamma$ :

$$
C C D F(\gamma)=\operatorname{Prob}(P A P R>\gamma) .
$$

Consider the OFDM system whose transmitter power amplifier is designed to allow PAPR up to $\gamma_{o}$. Then, a clipping does not occur as long as PAPR does not exceed $\gamma_{o}$, and the clipping probability is given by $C C D F\left(\gamma_{o}\right)$.

Motivated by this, we propose a simple early termination algorithm for SLM: (1) in the region where clipping does not occur in the conventional SLM (i.e., $P A P R \leq \gamma_{o}$ ), the proposed scheme terminates searching process immediately when it meets a candidate OFDM symbol whose PAPR is less than a pre-determined target PAPR threshold value; (2) otherwise, it performs a FS like the conventional SLM. The flowchart of the proposed algorithm is shown in Fig. 2, where $T H$ represents the target PAPR threshold. Note that $T H$ should be chosen not to increase the clipping probability, i.e., $T H \leq \gamma_{o}$. Also, the probability of performing a FS in the proposed scheme is equal to $C C D F(T H)$.

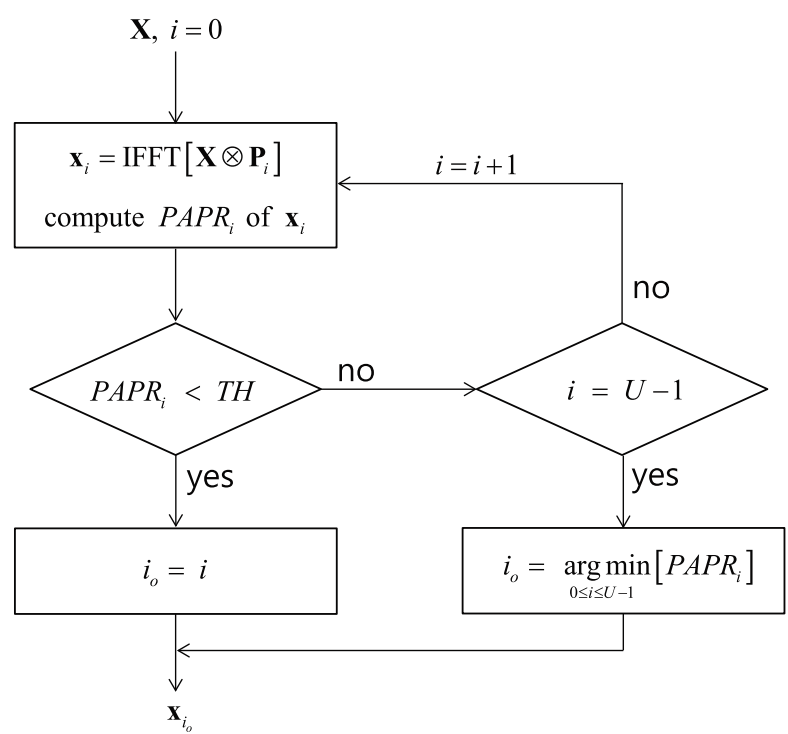


The transmitter power amplifier is generally designed such that the clipping probability, $\operatorname{CCDF}\left(\gamma_{o}\right)$ is very low. Thus, if we set $T H=\gamma_{o}$, the probability of performing a FS in the proposed scheme also becomes very low; that is, the early termination will occur for most OFDM symbols, which results in reduction of computational complexity. Of course, by adjusting $T H$, we can control the computational complexity of the proposed scheme.

\section{Simulation results}

For performance comparison, we consider the SLM-based OFDM system with $N=128$ where 16-QAM is used for a digital modulation. $U(\in\{4,16,64\})$ phase sequences are used: in order to compute $\left\{\mathbf{X} \otimes \mathbf{P}_{i}\right\}$ without complex multiplication,

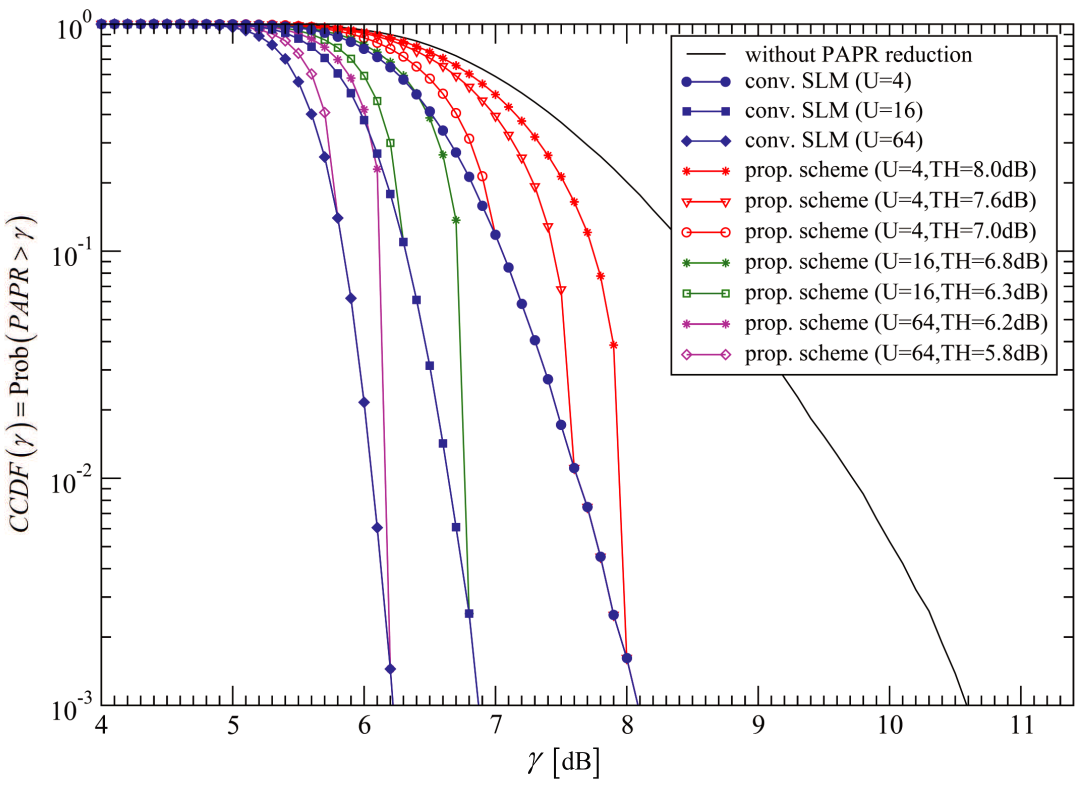

(a)

\begin{tabular}{|c|c|c|c|c|}
\hline & $T H[\mathrm{~dB}]$ & $C C D F(T H)$ & $\bar{N}_{P A P R}$ & $\bar{N}_{P A P R} / U$ \\
\hline \multirow{3}{*}{$U=4$} & 8.0 & $1.61 \times 10^{-3}$ & 1.243 & 0.311 \\
\cline { 2 - 5 } & 7.6 & $1.11 \times 10^{-2}$ & 1.470 & 0.368 \\
\cline { 2 - 5 } & 7.0 & $1.18 \times 10^{-1}$ & 2.141 & 0.535 \\
\hline \multirow{3}{*}{$U=16$} & 6.8 & $2.53 \times 10^{-3}$ & 3.121 & 0.195 \\
\cline { 2 - 5 } & 6.3 & $1.10 \times 10^{-1}$ & 6.872 & 0.430 \\
\hline \multirow{3}{*}{$U=64$} & 6.2 & $1.45 \times 10^{-3}$ & 9.800 & 0.153 \\
\cline { 2 - 5 } & 5.8 & $1.40 \times 10^{-1}$ & 28.212 & 0.441 \\
\hline
\end{tabular}

(b)

Fig. 3. (a) CCDF comparison between the conventional SLM and the proposed scheme ( $N=128$ and 16-QAM modulation). (b) Computational complexity of the proposed scheme. 
phase sequences whose each symbol $P_{i}(k)$ has the value in $\{ \pm 1, \pm j\}$ are generated randomly with a uniform distribution [5].

As a measure of computational complexity, we consider the number of PAPR computation which includes phase sequence multiplication, $N$-point IFFT, and the operation of (2). Let us define $\bar{N}_{P A P R}$ as the average number of PAPR computation per OFDM symbol. Since $\bar{N}_{P A P R}$ is equal to $U$ for the conventional SLM scheme, $\bar{N}_{P A P R} / U$ indicates the ratio of the computational complexity of the proposed scheme to that of the conventional SLM.

Figure 3 (a) shows the CCDFs of the conventional SLM and the proposed scheme according to the value of $U$, and the computational complexity of the proposed scheme is summarized in Fig. 3 (b). Here, we assume that the transmitter power amplifier for the conventional SLM-based OFDM system was designed such that the clipping probability is less than 0.001 . Thus, $\gamma_{o}$ has the value of about $8.1 \mathrm{~dB}, 6.9$ $\mathrm{dB}$, and $6.2 \mathrm{~dB}$ for $U=4, U=16$, and $U=64$ cases, respectively, and the threshold value of the proposed scheme, $T H$ was chosen satisfying $T H \leq \gamma_{o}$.

First, in the case where $T H$ is set closely to $\gamma_{o}$ (in Fig. 3 (a), $T H=8.0 \mathrm{~dB}$ for $U=4, T H=6.8 \mathrm{~dB}$ for $U=16$, and $T H=6.2 \mathrm{~dB}$ for $U=64$ ), the proposed scheme reveals steeper slope around $T H$ : in the region of $P A P R<T H$, the computation load is significantly reduced due to the early termination; otherwise, a FS is performed like the conventional SLM in order to guarantee the clipping probability to be less than 0.001 . It is interesting to note that the computational complexity was reduced to less than $20 \%$ of that of the conventional SLM for $U=16$ and $U=64$ cases (refer to Fig. 3 (b)).

In addition, as we decrease $T H$, the CCDF curve of the proposed scheme gets closer to that of the conventional SLM, but the computational complexity increases. Specifically, in the case where $T H$ is chosen such that $C C D F(T H)$ has the value of about 0.1 (in Fig. 3 (a), $T H=7.0 \mathrm{~dB}$ for $U=4, T H=6.3 \mathrm{~dB}$ for $U=16$, and $T H=5.8 \mathrm{~dB}$ for $U=64)$, the CCDF performance of the proposed scheme is comparable to that of the conventional SLM, while using about half computations.

\section{Conclusion}

In an attempt to reduce the computational complexity of SLM-based PAPR schemes using a full search, an early termination algorithm was proposed. It terminates searching process immediately when PAPR goes down below the target PAPR threshold, $T H$ which was chosen not to increase the clipping probability of the transmit power amplifier.

Simulation results indicate that the proposed scheme can control the trade-off between the CCDF performance and the computational complexity. Specifically, (1) the computation load can be minimized by setting $T H$ closely to $\gamma_{o}$, while maintaining the clipping probability of the conventional SLM; (2) by setting $T H$ such that $C C D F(T H) \approx 0.1$, we can obtain the CCDF performance comparable to that of the conventional SLM, with about half the amount of computation. 


\section{Acknowledgments}

This work was supported by Hankuk University of Foreign Studies Research Fund of 2020 . 